\title{
Modeling Based on LS-SVM Method and Predicting Dewatering Ratio in Sludge Process
}

\author{
Fei Luo ${ }^{1, a}$, Xinghong Qiao1, b, Weihao Liao1,c \\ ${ }^{1}$ Key Laboratory of Autonomous Systems and Networked Control, Ministry of Education \\ Institute of Automation Science and Engineering, South China University of Technology \\ Guangzhou 510640, P.R. China \\ aaufeiluo@scut.edu.cn, bqiaoxinghong@126.com, cauliaowh@mail.scut.edu.cn
}

Keywords: Sludge Process; Discharging Sludge; Thickening and Dewatering; Least Square Support Vector Machine; Soft Sensor

Abstract. The sludge produced by treating the urban sewage needs thickening and dewatering and it is important for improving environment to reduce the quantity of discharging sludge by increasing dewatering ratio in sludge process. This paper proposes a novel method that can model least squares support vector machines (LS-SVM) by Soft-sensing to meet the characteristics of multivariable, nonlinearity, large time delay, and time-varying in sludge thickening and dewatering process, and predict the dewatering ratio. The results indicate that the proposed method can not only improve prediction accuracy, but also efficiently get high dewatering ratio by controlling thickening chemicals comparing to the cases using different methods.

\section{Introduction}

In sludge process, increasing the dewatering ratio of sludge to reduce the quantity of the discharging sludge has become a focus of environmental departments, which can reduce the for the environment pollution of cities. It is widely applied to measure the dewatering ratio of sludge by Micro-X-ray Analysis [1]. Currently available method for controlling the dewatering ratio of sludge is that the dewatering ratio of sludge is used as feedback data for building control models, based on which the thickening amount as the input of the sludge process is controlled. But it is very difficult to get good controlling effectiveness because the system of sludge process is that of multivariable, nonlinearity, large time delay, and time-varying. A rapid and convenient technique for modeling and predicting for sludge process becomes desirable basis for controlling the dewatering ratio of sludge.

A data-derived soft-sensor was proposed to predict the quality of sludge process under the existing information. In the last several years, the different algorithms such as artificial intelligence (AI) techniques [2-11] have become one of the most important topics in predicting the quality of sludge process. AI techniques produce better results than classic regression methods for developing the software sensors [2]. Under this circumstance, soft-sensor based on AI techniques gradually becomes the hot area for predicting the quality of sludge process. K. Sridevi et al. proposed a Back propagation neural network (BP-NN) model to provide accurate predictions of the quality of sludge process [6]. In another work by Han Hong-Gui et al., a recurrent self-organizing neural network was used to predict the quality of sludge process [7]. Artificial neural network (ANN), which is suitable for modeling the systems with multivariable, nonlinearity, large time delay, and time-varying such as sludge process, is used to model the sludge process and predict the quality of it [8-11]. They concluded that ANN as the one of soft-sensing methods was capable to model accurately the sludge process. Majid Bagheri et al. developed a hybrid artificial neural networks-genetic algorithm 
approach to improve the accuracy of the model and the prediction of the quality of sludge process by optimizing the key parameters of ANN [5].

As mentioned above, ANN and the related techniques have become one of the most important topics in the sludge process. The techniques have been recognized as powerful tools for the case of the non-linear system. However, these algorithms all have their own defects. Their main shortcoming of the techniques is the slowly training speed. To some extent, the defects constrain the application of the algorithm. In this paper, we propose an evolving approach for modeling the sludge process and predicting the quality of it by least squares support vector machines (LS-SVM). The remainder of this paper is organized as follows. In the next section, the main aspects of LS-SVM model are briefly illustrated. Then the LS-SVM gives reasonable estimates for modeling the sludge process and predicting the dewatering ratio of it and comparison of three different methods in Section 3. The concluding remarks are presented in the last section.

\section{LS-SVM Algorithm}

In machine learning theory, Support Vector Machines (SVM) is a powerful methodology for providing better prediction results [12]. However, this algorithm is likely to consume too much of memory and computation time, due to the quadratic programming (QP) problem with linear constrain of the SVM formulation. Least Squares Support Vector Machines (LS-SVM) are standardized version of SVM, it works with the quadratic programming problem by replacing the inequality constraints with a linear Karush-Kuhn-Tucker system [13]. The basic formulation of LS-SVM is described as follows. Consider a given regression dataset of the form: $\left(x_{i}, y_{i}\right), i=1,2, \cdots, N, x_{i} \in R^{n}$ and $y_{i} \in R$ represent the input and output data, respectively, the regression model is taken the form

$$
y(x)=\omega^{T} \varphi(x)+b \quad \omega \in H, b \in R
$$

where the kernel function $\varphi\left({ }^{*}\right)$ is a nonlinear mapping function that maps the input space into a higher dimensional feature space, $b$ is the bias term and $\omega$ is the weight vector. In Least Squares Support Vector Machines algorithm, the cost function $l$ is minimized by formulating the following optimization problem

$$
\begin{array}{ll}
\min _{\omega, e} J(\omega, e)=\frac{1}{2} \omega^{T} \omega+\frac{\theta}{2} \sum_{i=1}^{N} e_{i}^{2} & \theta>0 \\
\text { subject to } y_{i}=\omega^{T} \varphi\left(x_{i}\right)+b+e_{i} & i=1,2, \cdots, N
\end{array}
$$

where the parameter $\theta$ is the penalty weight controlling the overfitting phenomenon and the model complexity. $e_{i}=y_{i}-\tilde{y}_{i}$ is the regression error, and $e=\left[e_{i}, \cdots, e_{N}\right]$ is the learning residual vector. The first and second part of Eq. 2 present the weight decay and the evaluation of the accuracy of LS-SVM model for all training data. Eq. 3 gives the definition of the regression error. To solve this convex optimization problem, the following Lagrangian function is formed as [14, 15]:

$$
L(\omega, b, e, \alpha)=J(\omega, e)-\sum_{i=1}^{N} \alpha_{i}\left\{\omega^{T} \varphi\left(x_{i}\right)+b+e_{i}-y_{i}\right\}
$$


where $\alpha_{i} \in R, i=1,2, \cdots, N$ with Lagrange multipliers that can be positive or negative in the LS-SVM formulation. The optimum solution for Eq. 4 can be determined by the following set of partially differentiating equations,

$$
\begin{aligned}
& \frac{\partial L}{\partial \omega}=0 \Rightarrow \omega=\sum_{i=1}^{N} \alpha_{i} \varphi\left(x_{i}\right) \\
& \frac{\partial L}{\partial b}=0 \Rightarrow \sum_{i=1}^{N} \alpha_{i}=0 \\
& \frac{\partial L}{\partial e_{i}}=0 \Rightarrow \alpha_{i}=\theta e_{i} \quad i=1,2, \cdots, N \\
& \frac{\partial L}{\partial \alpha_{i}}=0 \Rightarrow \omega^{T} \varphi\left(x_{i}\right)+b+e_{i}-y_{i}=0
\end{aligned}
$$

It can be notice from the condition $\alpha_{i}=\theta e_{i}$ that the sparseness is lost. After eliminating variables $\omega$ and $e_{i}$, the solution can be obtained from the following linear equations.

$$
\phi\left[\begin{array}{l}
b \\
\alpha
\end{array}\right]=\left[\begin{array}{l}
0 \\
y
\end{array}\right]
$$

where $\phi$ is a square matrix given by:

$$
\phi=\left[\begin{array}{cc}
0 & 1_{n}^{T} \\
1_{n} & K+\theta^{-1} \mathrm{I}
\end{array}\right]
$$

where $y=\left[\begin{array}{llll}y_{1} & y_{2} & \cdots & y_{N}\end{array}\right]^{T}, \quad \alpha=\left[\begin{array}{llll}\alpha_{1} & \alpha_{2} & \cdots & \alpha_{N}\end{array}\right]^{T}, 1_{n}=\left[\begin{array}{llll}1 & 1 & \cdots & 1\end{array}\right]^{T}, \quad I$ is the $N \times N$ identity matrix, $K$ denotes the kernel matrix with $i j$ th element.

$$
K\left(x_{i}, x_{j}\right)=\varphi\left(x_{i}\right)^{T} \varphi\left(x_{j}\right)=\exp \left(-\frac{\left\|x_{i}-x_{j}\right\|}{2 \delta^{2}}\right) \quad i, j=1,2, \cdots, N
$$

where $\delta$ is the kernel size.

The parameters $\alpha$ and $b$ can be calculated by solving Eq. 12 as follows:

$$
\left[\begin{array}{l}
b \\
\alpha
\end{array}\right]=\phi^{-1}\left[\begin{array}{l}
0 \\
y
\end{array}\right]
$$

The resulting LS-SVM model for function estimation can get the following form:

$$
y(x)=\sum_{i=1}^{N} \alpha_{i} K\left(x, x_{i}\right)+b
$$


The structure diagram for the control design is shown in Figure 1. The output of this simple usage can be obtained by using Eq. 13 .

$$
\bar{y}=\sum_{i=1}^{M} y_{i}
$$

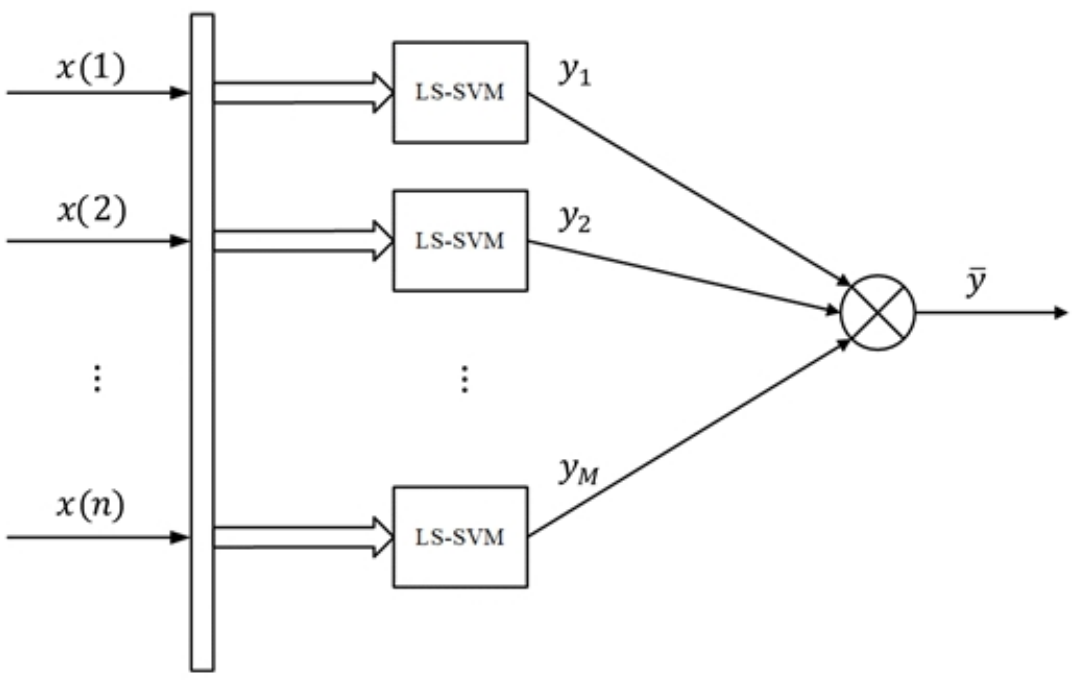

Figure 1 The structure of LS-SVM method

\section{Simulation and Results Analysis}

The LS-SVM algorithm mentioned above is put in use for a prediction of dewatering ratio of sludge processed by thickening and dewatering from a wastewater treatment plant. The dataset used in this study is supplied by a sludge process company. A total of 527 daily data records from a plant processing sludge have been used, each consisting of 7 processing variables, which are measured in different time points. After cleaning some missing values, principal component analysis (PCA) is performed for the remaining data points. The aim of performing PCA is to select the only relevant variables.

The input variables $X$ of the soft-sensor model consist of 6 thickening variables such as lime et al. The model's output variable y is the concentration of dewatering ratio of sludge.

The performance of the proposed approach is evaluated by using root mean square error (RMSE) method.

$$
R M S E=\sqrt{\sum_{i=1}^{K}\left(\bar{y}_{i}-y_{i}^{*}\right)^{2} / K}
$$

where $\bar{y}_{i}$ is the predicted value of our model, $y_{i}^{*}$ is the corresponding true value from the dataset, and $K$ denotes the size of the dataset.

70 data in the relevant variables are used to train model and the other 330 data are used to test model. The Table 1 indicates the comparison the ANN, SVM, LS-SVM based soft-sensor models. The RMSE of LS-SVM is 0.1088, which is smaller compared to that of the other two soft-sensor models. The LS-SVM algorithm has a better approximation performance in prediction of dewatering ratio of sludge than the other two methods. The experimental results demonstrate the 
effectiveness and reliability of the proposed method, which can be used to control the quantity of thickening chemicals to get high dewatering ratio that is good for improving environment.

Table 1 The performances of the three algorithms

\begin{tabular}{c|c}
\hline Algorithm & RMSE \\
\hline ANN & 0.1801 \\
\hline SVM & 0.1562 \\
\hline LS-SVM & 0.1088 \\
\hline
\end{tabular}

\section{Conclusions}

In this work, we propose an approach to predict the dewatering ratio of sludge by modeling for the process of thickening and dewatering sludge based on LS-SVM, which can get better modeling performance. The LS-SVM is applied to predict dewatering ratio of sludge in a plant of processing sludge by the soft-sensor model. The results demonstrate that the proposed method can improve prediction, comparing to the cases using ANN and SVM.

\section{Acknowledgements}

This work was financially supported by Science and Technology Planning Project of Guangzhou (201604010032), and Science and Technology Planning Project of Guangdong Province (2016B090918028).

\section{References}

[1] Shiota Kenji et al, Cesium Speciation in Dust from Municipal Solid Waste and Sewage Sludge Incineration by Synchrotron Radiation Micro-X-ray Analysis. ANALYTICAL CHEMISTRY , 2015, 87(22):11249-11254

[2] Gagik Badalians Gholikandi et al, Optimizing stabilization of waste-activated sludge using Fered-Fenton process and artificial neural network modeling (KSOFM, MLP). Environ Sci Pollut Res, 2014, 21:7177-7186

[3] Yuankai Zhang et al, Simple model of sludge thickening process in secondary settlers. Frontiers of Environmental Science \& Engineering, 2016, 10(2): 319-326

[4] Sebastian Kujawa et al, Neural image analysis for maturity classification of sewage sludge composted with maize straw. Computers and Electronics in Agriculture, 2014, 109:302-310

[5] Majid Bagheri et al, Modeling and optimization of activated sludgebulking for a real wastewater treatment plant using hybrid artificial neural networks-genetic algorithm approach. Process Safety and Environmental Protection, 2015, 95:12-25

[6] K. Sridevi et al, Back propagation neural network modelling of biodegradation and fermentative biohydrogen production using distillery wastewater in a hybrid upflow anaerobic sludge blanket reactor. Bioresource Technology, 2014,165:233-240

[7] Han Hong-Gui et al, A soft computing method to predict sludge volume indexbased on a recurrent self-organizing neural network. APPLIED SOFT COMPUTING, 2016, 38:477-486 
[8] Mendes Carlos et al, Artificial Neural Network Modeling for Predicting Organic Matter in a Full-Scale Up-Flow Anaerobic Sludge Blanket (UASB) Reactor. ENVIRONMENTAL MODELING \& ASSESSMENT, 2015,20(6):625-635

[9] Huang Y. W. et al, Artificial neural network modeling of thin layer drying behavior of municipal sewage sludge. MEASUREMENT, 2015,73:640-648

[10] Yu Guang-ping et al, Research on prediction method of sludge bulking based on ANN and grey Markov model. 2015 IEEE International Conference on Cyber-Technology in Automation, Control and Intelligent Systems (CYBER), Shenyang China, 8-12 June 2015, pp. 1622-1627

[11] Keskitalo Jukka et al, Artificial Neural Network Ensembles in Hybrid Modelling of Activated Sludge Plant. 7th IEEE International Conference on Intelligent Systems(IEEE IS), Warsaw POLAND, 24-26 SEP 2014, pp. 683-694

[12] Gencoglu M T, Uyar M. Prediction of flashover voltage of insulators using least squares support vector machines. Expert Systems with Applications, 2009, 36(7): 10789-10798

[13] Suykens J A K, Vandewalle J. Least squares support vector machine classifiers. Neural processing letters, 1999, 9(3): 293-300

[14]Wei G, Li G, Wu Y, et al. Application of Least Squares-Support Vector Machine in system-level temperature compensation of ring laser gyroscope. Measurement, 2011, 44(10): 1898-1903

[15] Kisi O. Modeling discharge-suspended sediment relationship using least square support vector machine. Journal of hydrology, 2012, 456: 110-120 